\title{
Community perception on health messaging with Viber application on modifiable risk factors of non-communicable diseases: a qualitative study
}

\author{
Swe Mar Myint Lwin*, Khaing Lay Mon*, Aung Soe Htet** \\ *Department of Health Behavior and Communication, University of Public Health, Yangon, Myanmar \\ ** International Relations Division, Ministry of Health, Nay Pyi Taw, Myanmar/University of Oslo, Oslo, Norway \\ DOI: 10.29322/IJSRP.11.09.2021.p11710 \\ http://dx.doi.org/10.29322/IJSRP.11.09.2021.p11710
}

\begin{abstract}
In many countries around worldwide, mobile health technology is increasingly being used and it may be a convenient and costeffective way to support healthy behaviors for preventive health care. The present study aims at exploring the community perception on the health messaging intervention with the Viber application conducted in the rural population of Yangon, Myanmar. In-depth interviews were conducted among ten participants of those who improved any behavior or metabolic risk factors of noncommunicable disease (NCDs) and who did not change after the intervention. They were purposively selected according to the result of a previous community based quasi-experimental study with one-year intervention (from 2019 to 2020) of health messaging with Viber application in the rural area of Twantay Township, Yangon, Myanmar. As the result, all participants perceived the health messages were useful. Salient findings included that improving their health knowledge, a reminder on healthy behaviors, convenience in getting health information, reducing risky behaviors, and conveying the health message to their family members and friends as the major advantages. Time constraints, visual impairment, technical skill insufficiency, poor phone and internet connection, and privacy issues seemed to be the difficulties in reading health messages among the participants. Despite some difficulties in reading the health messaging, most of the participants perceived mobile phone health messaging as a convenience to them compared to face-to-face communication with health staff for getting health information of NCD risk factors.
\end{abstract}

Keywords: Perception, mobile health messaging, risk factors of NCDs

\section{INTRODUCTION}

The number of mobile phone usage was increasing in developing countries and short message services have become the new and innovative opportunities for disease prevention (1). The burden of non-communicable diseases (NCDs) mortality has risen rapidly in recent decades although many risk factors can be prevented (2). Like other low-middle income countries, Myanmar is encountering a surge burden of NCDs and their risk factors and stood at the higher rank among Southeast Asia countries(3). Effective preventive measures for NCD risk factors can attain a positive impact and substantial mitigation of NCD mortality in a population (4). Mobile health messaging could offer a convenient and cost-effective way to support desirable health behaviors for preventive health care (2). One of the feasible points was that there were 22 million internet users in Myanmar in January 2020, with the number of internet users increasing by 1.0 million (4.8 \%) between 2019 and 2020 (5). The majority of Myanmar internet users enjoy the Viber application as a user-friendly social platform in both urban and rural areas. Thus, evidence and strategies of mobile phone health messaging are needed to improve as innovative public health interventions in modern society. To assess the effect of mobile phone health messaging This publication is licensed under Creative Commons Attribution CC BY. 
International Journal of Scientific and Research Publications, Volume 11, Issue 9, September 2021

with the Viber application, we conducted a mixed-methods study including a quantitative survey and qualitative in-depth interviews in rural area of Twantay Township in 2019. The present study is the qualitative portion of the study that intended to explore the perception of the community on the mobile phone health messaging on risk factors of major NCDs in rural populations. A better understanding of the perception of the community on the mobile phone health messaging intervention will improve the effectiveness and advantages of the health messaging intervention with the Viber application, which might be one of the ways for the prevention of NCDs risk factors in Myanmar.

\section{METHODS}

Participants and study site

A community-based quasi-experimental study including a control group was conducted among 240 community members for a oneyear duration with the intervention of mobile phone health messaging with the Viber application in the rural area of Twantay Township, Yangon Region during 2019 and 2020 (6). Only the intervention group was being sent health messaging with the Viber application once a week by grouping according to their presence of respective risk factors of major NCDs. Health messages included both type of text and pamphlet photos officially distributed from Ministry of Health, Myanmar. Based on the study result, a total of ten participants from the intervention group were purposively selected for this qualitative study. Semi structures interview guideline was developed and participants were purposefully chosen to represent a diverse range of perspectives, including participants of various ages, genders, occupations, and status of changing/improving their any risk factors of major NCDs (smoking, smokeless tobacco, and alcohol drinking, increase BMI, high blood pressure, high level of fasting blood glucose and high level of blood lipid profile) after the one-year intervention period.

Data analysis

All interviews were transcribed and translated into English language and Atlas. ti (version 6.1) qualitative data analysis software was used to review each interview transcript and highlight relevant segments of the data (quotations) that were meaningful, informative, and impactful. A codebook was developed with code names and descriptions of codes and 40 codes were come out from all transcripts and all interviews were coded according to it in Atlas. ti software. Each coded quotation was reviewed to ensure that it was assigned to the appropriate code and then combined with similar codes to develop themes and subthemes. Thematic analysis was conducted and four main themes were developed from the interviews.

\section{RESULTS}

Most of the respondents were between the age of 41 to 60 years old, female, and attained below high school level of education in both intervention and control group (Table 1). The majority were the seller and own business person. The findings revealed four main themes (a) perception of participants on health education with Viber application; (b) advantages of mobile phone health messaging intervention; (c) difficulties on mobile phone health messaging intervention and (d) suggestions on finding ways to improve the intervention.

\section{Theme I: Perception of participants on health education with Viber application}

All participants perceived that receiving health messages was useful and they understood their rural population required a better level of health knowledge. Furthermore, no one had previous experience of receiving health education in a similar mobile application like Viber and therefore they regarded this as a new and innovative technique. The respondents expressed their appreciation on receipt of health text messages with the Viber application that increased the level of their knowledge on health. Besides, they could re-read the 
International Journal of Scientific and Research Publications, Volume 11, Issue 9, September 2021

ISSN 2250-3153

health messages whenever they want to read them. The following quotations reflected their perception of participants on the usefulness of Viber health messages.

"In my opinion, most of the community members in rural areas have a low level of health knowledge. By conducting this program, they can get more knowledge on health. A written text is worth a hundred words, and they can re-read the text in the Viber groups. A word or saying may be forgotten but text or written form may remain." (ID9)

"I think receiving health education in this way is effective and we come to know the things that we did not know previously from this program."(ID-2)

Four participants preferred receiving health education in person by health staff to the Viber application for the interactive two-way conversation with health staff that offered chance to explain the detail about the diseases. On the other hand, the rest preferred receiving health education with the Viber group in order to avoid a time-consuming appointment with health staff, and health messages were easily accessible on their mobile. The following extracts showed their aspects of preference on the method of receiving health education.

\section{"We can interact with health personals during the health education program in person but in the Viber} group, we can just read and are unable to respond."(ID-8)

"I prefer reading the text of Viber message to receiving health education in person because reading does not take time but meeting in person with health staff takes more time."(ID-7)

Comparing texts and photo messages, most of the participants preferred text messages rather than photo messages as the texts written in the photos were too small to read clearly, while one participant showed his preference of photo message that was more interesting. The following excerpts pointed out their preference for the type of message.

"For me, reading text is more preferable. The text in pictures was too small to read for me. Therefore I like reading text, and I can see the text in the Viber App." (ID-9)

"I prefer text messaging because I can listen to and see the texts when they (family member) are reading it for me and I can follow these instructions. " (ID-8)

\section{Theme II: Advantages of mobile phone health messaging with Viber application}

Improving health knowledge, as a reminder on healthy behaviors, and convenience in getting health information, reducing risk behaviors of NCDs, and conveying the health message they received to others were mentioned as advantages in the interviews. Most respondents stated that they had gained awareness and improved the level of health knowledge on healthy lifestyle, especially in the 
International Journal of Scientific and Research Publications, Volume 11, Issue 9, September 2021

areas of healthy diet, hazards of alcohol drinking, smoking, and smokeless tobacco consumption by participating in the intervention group. The following quotations illustrated their outlooks.

"I had more knowledge concerning diet patterns suitable for diabetes patients and the consequences of alcohol drinking and smoking from health messages."(ID-9)

"Although I already know about some information from health messages, most were new for me, and then, I have changed my diet and lifestyle after involving this Viber group as a participant."(ID-4)

Four respondents recognized that sending regular health messaging reminded them to keep healthy behaviors. Although the respondents already knew some facts about a healthy diet, they overlooked to behave in practice. The received health messages alerted the respondents on how to live a healthy lifestyle.

"Although we forget something concerning a healthy lifestyle, we can read text messages you sent and remind me to remember to reduce salt and oil consumption and to carry out physical exercise. ”(ID-7)

Some of the respondents answered that reading health messages with the Viber groups was a convenient way of getting health information than other ways. They mentioned that they did not use to watch health programs on television purposively. Instead, the respondents could access online health information with the Viber application that was automated over the mobile phone.

“As we didn't used to watching television programs for health information, we cannot catch the information transmitted from TV every time. But receiving health messages from the Viber is convenient because we can easily see them after just opening the phone and the internet." (ID-4)

Some said that after the intervention, they had changed to a healthy diet lifestyle such as having more vegetables and fruits, reducing oil, and salt consumption during cooking. One participant said that he quit smoking and alcohol drinking last two months ago after reading the health messages with the Viber group.

"My family consumed cooking oil a lot previously and I become worried about oil consumption and prefer more fruit and vegetable after knowing about health messages. " (ID-5)

"I used to smoke 4 or 5 cigarettes per day and drink alcohol about one-third of cups daily, however, Since two months ago, I quit them after getting health knowledge from Viber health messages." (ID-2)

Three participants conveyed the health messages received from the Viber application on diet patterns especially to increase intake of more fruit and vegetable and reduce the cooking oil and salt consumption to their family members, relatives, and friends.

"I convey the Viber health message about how to cook healthy and to increase intake of more fruit and vegetables to my family members who live away from me."(ID-4) 
"Concerning health messages, I use to relay health information to my family particularly to my

daughter to reduce oil in cooking and reduce salt intake not to be hypertension." (ID-2)

\section{Theme III: Difficulties on mobile phone health messaging with Viber application}

The respondents mentioned time constraints, visual impairment, technical skill insufficiency, poor phone and internet problems, and privacy issues as their difficulties in reading health messaging with Viber application and practicing according to the health messages.

Most participants couldn't follow the health instructions from health messages especially in physical activity and a healthy diet due to time constraints. The following extracts highlighted this issue.

"Although I want to carry out the physical exercise, I cannot do that because I hardly have some free time in afternoons."(ID-3)

"Although I know that following the instructions of health messages like plate model for a healthy diet can be effective for health, we can't follow detail about that because of our struggling lifestyle and condition."(ID-9)

Two participants found some difficulties in reading the health message due to the small font size of text messages on the phone screen but could read with glasses.

"As the font size is small, I can't see them clearly and I need glasses for reading."(ID-9)

Three participants needed help from their family members to read because of the insufficiency of technical skills on the mobile phone and the internet.

"I cannot manage it but my niece read the health message for me due to a lack of technical skill on the mobile phone."(ID-3)

Two respondents complained about the poor internet connection in the study area, Twantay Township, while the remaining participants were satisfied with their internet connection.

"As a difficulty, it happened when the internet connection was weak and temporary broken down of mobile phone because my children played with the phone."(ID-7)

A respondent pointed out the privacy issue in the Viber group.

"I prefer asking questions that I want to know directly in the Viber group but I did not because I think it needs more privacy and I afraid my questions to be seen by other people in the group."(ID-9) 
International Journal of Scientific and Research Publications, Volume 11, Issue 9, September 2021 ISSN 2250-3153

\section{Theme IV: Suggestion on finding ways to improve the intervention}

To improve giving health education with the Viber application, the respondents advised on the frequency of health messaging and selection of age group for future intervention plans with Viber health messaging.

Almost all of the respondents stated that sending Viber messages weekly was convenient except one, who suggested increasing the frequency twice a week.

"It will be better to be sent health messages twice a week because sometimes I forget about the messages as I am busy with my housework and chores of my children only if sending once a week." (ID-7)

Most respondents suggested recruiting older age groups and those who had the disease because older age group might interest more to participate in the Viber health messaging group. On the other hand, two of them suggested selecting the younger age groups because older ages might have eye problems and technical problems in reading messages although they were interested in the health messages. The following examples expressed their suggestions.

"If the participant had any disease, he will be more interested in the intervention than participants who had no underlying disease."(ID-9)

"If this intervention is conducted in other places, you should select older people to involve in the Viber group, because if you chose younger people, they may not have any diseases. "(ID-2)

\section{DISCUSSION}

Using mobile health technologies for improving population-level health outcomes around the world was emerged in the last decade (7). The present study findings showed that all selected participants perceived as sending health messages with the Viber group were effective and new for them. Similar to the figures from other studies conducted in Palestine, Lebanon, and Latin America, where participants expressed their interest in mobile health (mHealth) and they enjoyed this kind of experience (1) (8) (9). Different preferences for receiving health education methods were found out in the present study. More than half of the participants preferred receiving health education with the Viber application because they thought that planning to meet with health staff to receive health information was time-consuming than reading messages with Viber. On the other hand, some preferred receipt of health education in person by health staff because they could have a chance to ask more questions to health staff. According to a similar finding in rural Bangladesh (10), some participants did not see the value of using mobile phones for healthcare when compared to face-to-face consultations. According to the interviews, receiving health messages from Viber improved their health information accessibility and facilitating self-management and this finding was consistent with a systematic review of qualitative studies on patient's perception of mHealth application (11). According to their responses, being sent health messages weekly made them a reminder of a healthy lifestyle, and they expressed satisfaction with being reminded of important health-related issues which are consistent with the finding from Lebanon (8). Similar findings with a study conducted in Lebanon (8), participants conveyed the health message concerning diet patterns to their family members, relatives, and friends and this could be the advantage of mobile health messaging intervention. Almost all participants could not follow the instruction from health messages concerning physical exercise and their main reason was the limitation of their free time for physical exercise. The technology gap was one of the mHealth barriers in improving health outcomes (12) (10) and they also mentioned that poor technical skills on phones and the internet were one of the difficulties for them 
International Journal of Scientific and Research Publications, Volume 11, Issue 9, September 2021

especially in older ages. Following their responses, the internet connection was not a problem in the Twantay Township except for a slow connection for some participants during the intervention period. In a meta-ethnographic review of qualitative studies, the privacy issue was pointed out and which is similar to the current study (11). By evaluation the perception of the respondents involved in the one-year intervention of health messaging with the Viber application, we could consider using this way as an add-in method of reducing risk factors of major NCDs in our country.

\section{CONCLUSION}

In conclusion, all respondents accepted the usefulness of the health messaging intervention with the Viber application. Although some favored the method of receiving health education with Viber application, some still preferred receiving health education in person.

\section{DECLARATIONS}

Funding: The present research was funded by an implementation research grant by the Ministry of Health, Myanmar.

Conflict of interest: No conflict of interest

Ethical approval: This study received ethical approval from the Institutional Review Board of the University of Public Health, Yangon, Myanmar with IRB number [UPH-IRB (2019/IR Research/22)].

\section{ACKNOWLEDGEMENTS}

The authors gratefully acknowledge all professors and senior colleagues from the University of Public Health, Yangon for their enormous support of this research.

\section{REFERENCE}

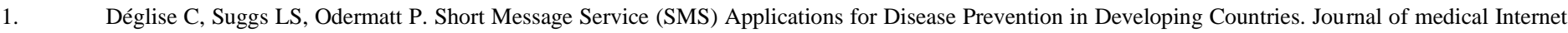
research. 2012 Jan 12;14:e3.

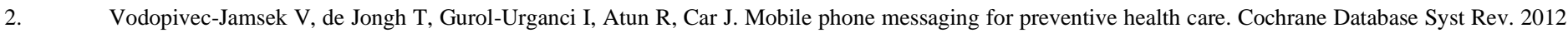
Dec 12;12:CD007457.

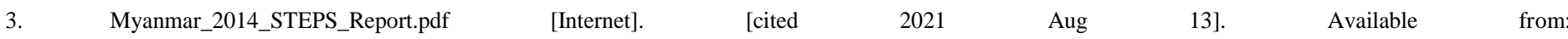
https://www.who.int/ncds/surveillance/steps/Myanmar_2014_STEPS_Report.pdf

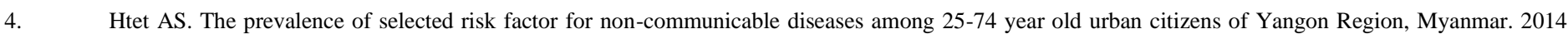
[cited 2021 Jul 1]; Available from: https://www.duo.uio.no/handle/10852/44123

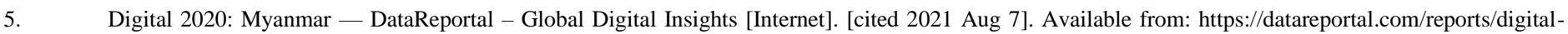
2020-myanmar

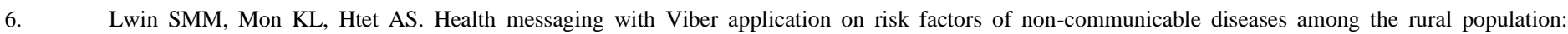

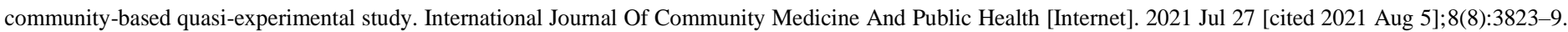
Available from: https://www.ijcmph.com/index.php/ijcmph/article/view/8310

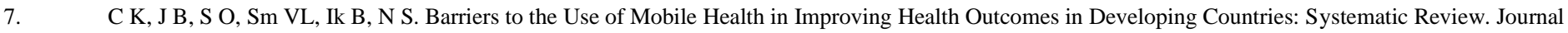
of medical Internet research [Internet]. 2019 Oct 9 [cited 2021 Jun 18];21(10). Available from: https://pubmed.ncbi.nlm.nih.gov/31593543/ 


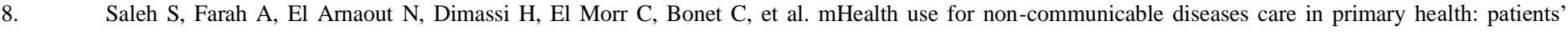
perspective from rural settings and refugee camps. Journal of public health (Oxford, England). 2018 Oct 11;40.

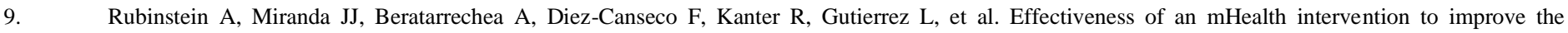

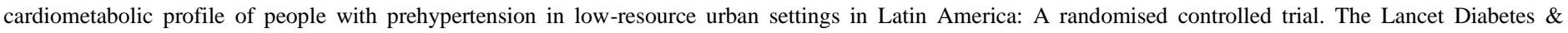
Endocrinology. 2015 Dec 1;4.

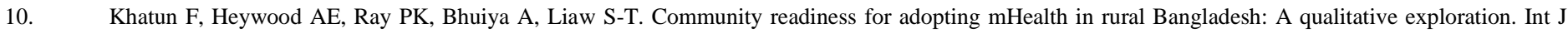
Med Inform. 2016 Sep;93:49-56.

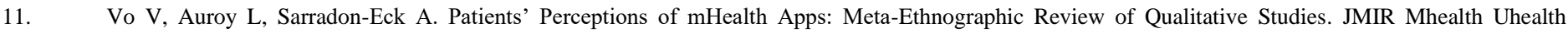
[Internet]. 2019 Jul 10 [cited 2021 Jul 1];7(7):e13817. Available from: https://www.ncbi.nlm.nih.gov/pmc/articles/PMC6652126/

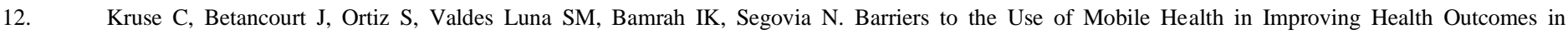
Developing Countries: Systematic Review. J Med Internet Res. 2019 Oct 9;21(10):e13263.

\section{AUTHORS}

First Author -Dr.Swe Mar Myint Lwin, Lecturer, Department of Health Behavior and Communication, University of Public Health Yangon, email: swemarmyintlwin@gmail.com

Second Author - Dr.Khaing Lay Mon, Professor and Head, Department of Health Behavior and Communication, University of Public Health Yangon, email: drlaymonuph@gmail.com

Third Author -Dr.Aung Soe Htet, Deputy Director, International Relations Division, Ministry of Health, Nay Pyi

Taw, Myanmar/ University of Oslo, Oslo, Norway, email: aungsh@gmail.com

Correspondence Author- Dr, Swe Mar Myint Lwin, email:swemarmyintlwin@gmail.com, Mobile:+9595126289 
Table I: Demographic characteristic of respondents in the study $(n=10)$

\begin{tabular}{|l|l|}
\hline Characteristics & Frequency \\
\hline Age (completed age in year) & \\
\hline $30-40$ & $\mathbf{2}$ \\
\hline $41-50$ & $\mathbf{4}$ \\
\hline $51-60$ & $\mathbf{4}$ \\
\hline Gender & \\
\hline Male & $\mathbf{3}$ \\
\hline Female & $\mathbf{7}$ \\
\hline Education & \\
\hline Below high school level & $\mathbf{7}$ \\
\hline High school level and above & $\mathbf{3}$ \\
\hline Occupation & \\
\hline Dependent & $\mathbf{1}$ \\
\hline Own Business/Seller & $\mathbf{6}$ \\
\hline Farmer & $\mathbf{2}$ \\
\hline Government Staff & $\mathbf{1}$ \\
\hline Status of risk factors after intervention & \\
\hline Changed & $\mathbf{5}$ \\
\hline Not changed & $\mathbf{5}$ \\
\hline
\end{tabular}

